\title{
Embeddings between Triebel-Lizorkin Spaces on Metric Spaces Associated with Operators
}

https://doi.org/10.1515/agms-2020-0120

Received August 31, 2020; accepted December 16, 2020

Abstract: We consider the general framework of a metric measure space satisfying the doubling volume property, associated with a non-negative self-adjoint operator, whose heat kernel enjoys standard Gaussian localization. We prove embedding theorems between Triebel-Lizorkin spaces associated with operators. Embeddings for non-classical Triebel-Lizorkin and (both classical and non-classical) Besov spaces are proved as well. Our result generalize the Euclidean case and are new for many settings of independent interest such as the ball, the interval and Riemannian manifolds.

Keywords: Besov spaces; distributions; doubling volume property; embeddings; heat kernel; metric spaces; Triebel-Lizorkin spaces

MSC: Primary: 58J35, 58J40, Secondary: 42B35, 42B25, 42B15, 46F10

\section{Introduction}

Function spaces play a leading role in analysis and its applications for almost a century. For a plenty of scopes the researcher may need to "count" the behavior of a function regarding its continuity, differentiability, integrability and so and so forth. The functions that enjoy a similar level of the preceding properties, build the corresponding function spaces.

Smoothness spaces have been initially introduced because of the straightforward need to measure the integrability of a function and its derivatives and have been further developed under the leverage of the Fourier transform.

Two of the most general scales, which are involved in a large number of applications, are Triebel-Lizorkin spaces and the companion class of Besov spaces. For the historical path of the development of function spaces, we refer to [30].

On the other hand, the problem under study may be governed by a geometry which is not the Euclidean one. For such a purpose significant progress in function spaces on several geometric settings -spheres, balls, manifolds and more- has been obtained the last decades [3, 4, 6, 19-21, 26, 31, 32].

Today the above function spaces have been introduced and explore in a very broad set-up that covers all the aforementioned cases [23].

Embeddings between various spaces of distributions play an important role in Function Theory with applications in PDE's, Approximation Theory and Statistics. Our goal in this article is to extend known results regarding the (Sobolev-type) embeddings of Triebel-Lizorkin and Besov spaces of different smoothness on $\mathbb{R}^{d}$ to the corresponding Classical and Nonclassical spaces associated to a non-negative self-adjoint operator $L$

${ }^{\star}$ Corresponding Author: Athanasios G. Georgiadis: Faculty of Engineering, Mathematics and Science, Trinity College, Dublin, Ireland, E-mail: georgiaa@tcd.ie

George Kyriazis: Department of Mathematics and Statistics, University of Cyprus, Nicosia, Cyprus, E-mail: kyriazis@ucy.ac.cy

Oopen Access. (ब) Commons Attribution alone 4.0 License. 
that allows as to deal with spaces with different geometries, compact and non-compact spaces and spaces with non-trivial weights.

The organization of the paper is as follows: In $\S 2$ we present the basic framework that will be needed as well as the definitions and the main assumptions of the various spaces. $\S 3$ contains the main result of the paper Theorem 3.1 which states that for $s_{0}, s_{1} \in \mathbb{R}, p_{0}, p_{1} \in(0, \infty)$ and $q, r \in(0, \infty]$ such that $s_{0}>s_{1}$ and $p_{0}<p_{1}$, then the Triebel Lizorkin space $F_{p_{0}, q}^{S_{0}}(L)$ is continuously embedded in $F_{p_{1}, r}^{S_{1}}(L)$ provided $\frac{s_{0}-s_{1}}{d}=$ $\frac{1}{p_{0}}-\frac{1}{p_{1}}$, where $d$ is the homogeneous dimension of the metric measure space. In $\S 4$ this result is extended to nonclassical Triebel-Lizorkin spaces in order to deal with anisotropic geometries. In section $\S 5$ we discuss similar results concerning classical and non classical Besov spaces. We note that the nonclassical spaces are of great interest and appear naturally in nonlinear approximation of sufficiently smooth disitributions by various decompositions systems such as frames (see [23] and the references therein for details). Finally in $\S 6$ we present a typical example that illustrates our results.

\section{Background}

This section provides the geometric setting we work on, some illustrative remarks and the necessary machinery for our study.

\subsection{The framework}

We are ready to present the main assumptions needed for our study.

Assumption $I$. We assume that $(\mathcal{M}, \rho, \mu)$ is a metric measure space such that $(\mathcal{M}, \rho)$ is locally compact with distance $\rho(\cdot, \cdot)$, and $\mu$ is a positive Radon measure satisfying:

(a) Doubling volume condition: There exists a constant $c_{0}>1$ such that

$$
0<|B(x, 2 r)| \leq c_{0}|B(x, r)|<\infty \text { for all } x \in \mathcal{M} \text { and } r>0,
$$

where $|B(x, r)|$ is the volume of the ball $B(x, r):=\{y \in \mathcal{M}: \rho(x, y)<r\}$.

(b) Noncollapsing condition: There exists a constant $c_{1}>0$ such that

$$
\inf _{x \in \mathcal{M}}|B(x, 1)| \geq c_{1}
$$

Assumption II. The geometry of the space $(\mathcal{M}, \rho, \mu)$ is related to a self-adjoint non-negative operator $L$ on $\mathbb{L}^{2}(\mathcal{M}, d \mu)$, mapping real-valued to real-valued functions, such that the associated semi-group $e^{-t L}, t>0$, consists of integral operators with (heat) kernel $p_{t}(x, y)$ obeying the conditions:

(c) Gaussian localization: There exist constants $c_{2}, c_{3}>0$ such that

$$
\left|p_{t}(x, y)\right| \leq \frac{c_{2} \exp \left\{-\frac{c_{3} \rho^{2}(x, y)}{t}\right\}}{[|B(x, \sqrt{t})||B(y, \sqrt{t})|]^{1 / 2}} \quad \text { for } x, y \in \mathcal{M}, t>0 .
$$

(d) Hölder continuity: There exists a constant $\alpha>0$ such that

$$
\left|p_{t}(x, y)-p_{t}\left(x, y^{\prime}\right)\right| \leq c_{2}\left(\frac{\rho\left(y, y^{\prime}\right)}{\sqrt{t}}\right)^{\alpha} \frac{\exp \left\{-\frac{c_{3} \rho^{2}(x, y)}{t}\right\}}{[|B(x, \sqrt{t})||B(y, \sqrt{t})|]^{1 / 2}}
$$

for $x, y, y^{\prime} \in \mathcal{M}$ and $t>0$, whenever $\rho\left(y, y^{\prime}\right) \leq \sqrt{t}$.

(e) Markov property:

$$
\int_{\mathcal{M}} p_{t}(x, y) d \mu(y)=1 \quad \text { for } x \in \mathcal{M} \text { and } t>0
$$


The setting considered in this paper has been put forward in $[7,23]$ and has been studied extensively in recent years $[1,2,5,9,10,15-18,22,24,25]$.

Note further that the doubling volume property is very classical in the literature starting by the celebrated work of Coifmann and Weiss [6]. Also the non-collapsing condition holds automatically on the Euclidean space and whenever the measure of the space is finite; $\mu(\mathcal{N})<\infty$, therefore it is restrictive only when $\mu(\mathcal{N})=$ $\infty$.

From (2.1) it follows that there exist constants $c_{0}^{\prime} \geq 1$ and $d^{\prime}>0$ such that

$$
|B(x, \lambda r)| \leq c_{0}^{\prime} \lambda^{d^{\prime}}|B(x, r)| \quad \text { for } x \in \mathcal{M}, r>0 \text { and } \lambda>1 .
$$

Definition 2.1 The minimum value $d>0$ of the above quantity $d^{\prime}$ will be called homogeneous or geometrical dimension of $(\mathcal{M}, \rho, \mu)$.

From (2.1) and (2.2), we extract

$$
|B(x, r)| \geq \frac{c_{1}}{c_{0}^{\prime}} r^{d} \quad \text { for } x \in \mathcal{M} \text { and } 0<r \leq 1 .
$$

The setting $(\mathcal{M}, \rho, \mu, L)$ is very general. A realization of it appears in the general framework of strictly local regular Dirichlet spaces with a complete intrinsic metric, see [7]. Some more examples of settings satisfying our assumptions are Riemannian manifolds with non-negative Ricci curvature, associated with the LaplaceBeltrami operator, Lie groups of polynomial volume growth with sub-laplacians, the Euclidean space $\mathbb{R}^{d}$, with uniformly elliptic divergence form operators, the weighted ball or the sphere with the corresponding Laplacians, the interval with Jacobi operators, the upper hemisphere and the simplex.

\subsection{Distributions}

For the definition of Besov and Triebel-Lizorkin spaces we follow Kerkyacharian and Petrushev [23] and introduce the notion of test functions and the corresponding distributions associated with $L$.

In the present setup, one has to distinguish between the two cases $\mu(\mathcal{M})<\infty$ and $\mu(\mathcal{M})=\infty$. We have the following.

- The case $\boldsymbol{\mu}(\mathcal{M})<\infty$. In this case we let $\mathcal{S}=\mathcal{S}(L)$ denote all functions $\phi \in \cap_{n} \mathcal{D}\left(L^{n}\right)$, where $\mathcal{D}\left(L^{n}\right)$ denotes the domain of the operator $L^{n}, n \geq 0$, with the topology induced by

$$
\mathcal{P}_{n}(\phi):=\left\|L^{n} \phi\right\|_{2}, \quad n \geq 0 .
$$

- The case $\boldsymbol{\mu}(\mathcal{M})=\infty$. The class of test functions $\mathcal{S}=\mathcal{S}(L)$ is defined as the set of all functions $\phi \in \cap_{n} \mathcal{D}\left(L^{n}\right)$ such that

$$
\mathcal{P}_{n, \ell}(\phi):=\sup _{x \in \mathcal{M}}\left(1+\rho\left(x, x_{0}\right)\right)^{\ell}\left|L^{n} \phi(x)\right|<\infty, \quad \forall n, \ell \geq 0 .
$$

Here $x_{0} \in \mathcal{M}$ is selected arbitrarily and fixed from now on. Note that the particular selection of $x_{0}$ in the above definition is not important.

Following a standard approach, the space $\mathcal{S}^{\prime}=\mathcal{S}^{\prime}(L)$ of distributions on $M$ is defined as the set of all continuous linear functionals on $\mathcal{S}$ and the pairing of $f \in \mathcal{S}^{\prime}$ and $\phi \in \mathcal{S}$ will be denoted by $\langle f, \phi\rangle:=f(\bar{\phi})$.

For further details on distributions in the present setting we refer the reader to [23]. 


\subsection{Spectral multipliers}

We recall that according to the spectral theorem, since $L$ is a non-negative self-adjoint operator, there exists a unique spectral resolution associated with $L$, consisted of orthoprojections on $L_{2}(M)$ such that

$$
L=\int_{0}^{\infty} \lambda d E_{\lambda} .
$$

Moreover, $L$ maps real-valued to real-valued functions and for any real-valued, measurable and bounded function $g$ on $\mathbb{R}_{+}$the operator (spectral multiplier) $g(L)$, defined by

$$
g(L):=\int_{0}^{\infty} g(\lambda) d E_{\lambda}
$$

is bounded on $L^{2}(M)$, self-adjoint, and maps real-valued functions to real-valued functions as well.

\subsection{Besov and Triebel-Lizorkin spaces}

In order to define Besov and Triebel-Lizorkin spaces, we need to consider the following auxiliary functions with the following properties.

Let the functions $\varphi_{0}, \varphi \in \mathfrak{C}^{\infty}\left(\mathbb{R}_{+}\right)$be such that

$$
\begin{aligned}
& \operatorname{supp} \varphi_{0} \subset[0,2], \varphi_{0}^{(2 v+1)}(0)=0 \text { for } v \geq 0,\left|\varphi_{0}(\lambda)\right| \geq c>0 \text { for } \lambda \in\left[0,2^{3 / 4}\right], \\
& \operatorname{supp} \varphi \subset[1 / 2,2],|\varphi(\lambda)| \geq c>0 \text { for } \lambda \in\left[2^{-3 / 4}, 2^{3 / 4}\right] .
\end{aligned}
$$

For now on we will denote by $\varphi_{k}(\lambda):=\varphi\left(2^{-k} \lambda\right)$ for $k \geq 1$. Having now fixed the couple $\left(\varphi_{0}, \varphi\right)$ we proceed to define the Triebel-Lizorkin spaces as in [23]:

Definition 2.2 Let $s \in \mathbb{R}, 0<p<\infty$, and $0<q \leq \infty$.

The Triebel-Lizorkin space $F_{p, q}^{s}=F_{p, q}^{S}(L)$ is defined as the set of all $f \in \mathcal{S}^{\prime}$ such that

$$
\|f\|_{F_{p, q}^{s}}:=\left\|\left(\sum_{k \geq 0}\left(2^{k s}\left|\varphi_{k}(\sqrt{L}) f(\cdot)\right|\right)^{q}\right)^{1 / q}\right\|_{L_{p}}<\infty
$$

with the $\ell^{q}$-norm replaced by the sup-norm when $q=\infty$.

It can be verified that the Triebel-Lizorkin spaces are independent from the admissible couple $\left(\varphi_{0}, \varphi\right)$, see [23]. Furthermore the preceding definition generalizes the standard definition of Triebel-Lizorkin spaces on $\mathbb{R}^{d}$, the sphere $\mathbb{S}^{d}$, the ball $\mathbb{B}^{d}$ and other settings.

\subsection{A Nikol'skii's type inequality}

A fundamental tool for the establishment of embedding theorems is Nikol'skii's inequality which, in its classical form on $\mathbb{R}^{d}$, relates different $L_{p}$-norms of band-limited distributions. In our setting the role of band-limited functions will be played by the spectral spaces:

Let $\lambda \geq 0$ and $Y$ a space of measurable function in $\mathcal{M}$. We denote by

$$
\Sigma_{\lambda}=\Sigma_{\lambda}(Y):=\left\{f \in Y: \theta(\sqrt{L}) f=f: \forall \theta \in \mathcal{C}_{0}^{\infty}(\mathbb{R}) \text { such that } \theta \equiv 1 \text {, in }[0, \lambda]\right\} .
$$

Note that for every $k \geq 0$ and every $f \in \mathcal{S}^{\prime}$ it holds that $\varphi_{k}(\sqrt{L}) f \in \Sigma_{2^{k+1}}$.

The following Nikol'skii's type inequality was proven in [24]: 
Proposition 2.3 Let $0<p \leq q \leq \infty$ and $\beta \in \mathbb{R}$. Then there exists a constant $c>0$ such that for any $f \in \Sigma_{\lambda}, \lambda \geq 1$,

$$
\left\|\left|B\left(\cdot, \lambda^{-1}\right)\right|^{\beta} f(\cdot)\right\|_{q} \leq c\left\|\left|B\left(\cdot, \lambda^{-1}\right)\right|^{\beta+\frac{1}{q}-\frac{1}{p}} f(\cdot)\right\|_{p} .
$$

We note that (2.15) holds even without assuming (2.2). In addition, if we apply (2.7) to (2.15) we easily get

$$
\left\|\left|B\left(\cdot, \lambda^{-1}\right)\right|^{\beta} f(\cdot)\right\|_{q} \leq c \lambda^{d\left(\frac{1}{p}-\frac{1}{q}\right)}\left\|\left|B\left(\cdot, \lambda^{-1}\right)\right|^{\beta} f(\cdot)\right\|_{p} .
$$

\section{Embeddings between Triebel-Lizorkin spaces}

We recall that in the case of Triebel-Lizorkin spaces defined on $\mathbb{R}^{d}$, it holds that when $-\infty<s_{1}<s_{0}<\infty$, $0<p_{0}<p_{1}<\infty$ and $0<q, r \leq \infty$, then $F_{p_{0}, q}^{s_{0}} \hookrightarrow F_{p_{1}, r}^{s_{1}}$, provided that $\frac{s_{0}-s_{1}}{d}=\frac{1}{p_{0}}-\frac{1}{p_{1}}$. Here we generalize this Sobolev embedding to the broad framework of this article.

Furthermore our results are new for many geometric settings of independent interest, such as the ball, the interval and Riemannian manifolds with nonnegative Ricci curvature and many more.

We are now ready to state and prove the main result of this paper.

Theorem 3.1 Let $s_{0}, s_{1} \in \mathbb{R}, p_{0}, p_{1} \in(0, \infty)$ and $q, r \in(0, \infty]$ be such that $s_{0}>s_{1}$ and $p_{0}<p_{1}$. Then

$$
F_{p_{0}, q}^{s_{0}} \hookrightarrow F_{p_{1}, r}^{s_{1}},
$$

provided that

$$
\frac{s_{0}-s_{1}}{d}=\frac{1}{p_{0}}-\frac{1}{p_{1}} .
$$

Proof. We need to prove that there exists $c>0$ such that for every $f \in F_{p_{0}, q}^{s_{0}}$,

$$
\|f\|_{F_{p_{1}, r}^{s_{1}}} \leq c\|f\|_{F_{p_{0}, q}^{s_{0}}} .
$$

Using the trivial embedding between the sequence spaces $\ell^{q} \hookrightarrow \ell^{\infty}$, it suffices to assume that $q=\infty$.

Let now $f \in F_{p_{0}, \infty}^{s_{0}}$ be such that $\|f\|_{F_{p_{0}, \infty}^{s_{0}}}=1$. Using that for every $p>0$ and $g \in L^{p}$

$$
\|g\|_{p}^{p}=p \int_{0}^{\infty} t^{p-1}|\{x \in \mathcal{M}:|g(x)|>t\}| d t,
$$

we can write

$$
\|f\|_{F_{p_{1}, r}^{s_{1}}}^{p_{1}}=p_{1} \int_{0}^{\infty} t^{p_{1}-1}\left|\left\{x \in \mathcal{M}:\left(\sum_{k=0}^{\infty}\left(2^{k s_{1}}\left|\varphi_{k}(\sqrt{L}) f(x)\right|\right)^{r}\right)^{1 / r}>t\right\}\right| d t .
$$

In order to estimate the summation in (3.4) from inequality (2.16) $\left(p_{0}<p_{1}\right)$ we get that for every $k \in \mathbb{N}_{0}$,

$$
\left\|\varphi_{k}(\sqrt{L}) f\right\|_{\infty} \leq c 2^{k d / p_{0}}\left\|\varphi_{k}(\sqrt{L}) f\right\|_{p_{0}} \leq c 2^{k\left(\frac{d}{p_{0}}-s_{0}\right)}\|f\|_{F_{p_{0}, \infty}^{s_{0}}}=c 2^{k\left(\frac{d}{p_{0}}-s_{0}\right)} .
$$

Let now $K \in \mathbb{N}_{0}$, employing (3.5) we find a constant $C^{\star}:=C^{\star}\left(p_{1}, p_{0}, r\right)>0$, such that

$$
\sum_{k=0}^{K}\left|2^{k s_{1}} \varphi_{k}(\sqrt{L}) f(x)\right|^{r} \leq c \sum_{k=0}^{K} 2^{k\left(\frac{d}{p_{0}}-s_{0}+s_{1}\right) r}=c \sum_{k=0}^{K} 2^{k \frac{d}{p_{1}} r} \leq C^{\star} 2^{K \frac{d}{p_{1}} r},
$$

where we also used assumption (3.2). On the other hand to estimate the upper sum of the series since $s_{1}-s_{0}<$ 0 , we have that for any integer $K \geq-1$

$$
\sum_{k=K+1}^{\infty}\left|2^{k s_{1}} \varphi_{k}(\sqrt{L}) f(x)\right|^{r} \leq c 2^{K\left(s_{1}-s_{0}\right) r} \sup _{k \geq 0}\left|2^{k s_{0}} \varphi_{k}(\sqrt{L}) f(x)\right|^{r} .
$$


Using (3.4) we write

$$
\begin{aligned}
\|f\|_{F_{p_{1}, r}^{s_{1}}}^{p_{1}} & =p_{1}\left(\int_{0}^{\left(2 C^{*}\right)^{\frac{1}{r}}}+\int_{\left(2 C^{*}\right)^{\frac{1}{r}}}^{\infty}\right) t^{p_{1}-1}\left|\left\{x \in \mathcal{M}:\left(\sum_{k=0}^{\infty}\left(2^{k s_{1}}\left|\varphi_{k}(\sqrt{L}) f(x)\right|\right)^{r}\right)^{1 / r}>t\right\}\right| d t \\
& =: p_{1}\left(I_{1}+I_{2}\right) .
\end{aligned}
$$

We first bound $I_{1}$. Applying (3.7) with $K=-1$ we derive

$$
\begin{aligned}
I_{1} & \leq c \int_{0}^{\left(2 C^{*}\right)^{\frac{1}{r}}} t^{p_{1}-1}\left|\left\{x \in \mathcal{M}: \sup _{k \geq 0}\left|2^{k s_{0}} \varphi_{k}(\sqrt{L}) f(x)\right|>t / c\right\}\right| d t \\
& \leq c \int_{0}^{c^{\prime}} t^{p_{0}-1}\left|\left\{x \in \mathcal{M}: \sup _{k \geq 0}\left|2^{k s_{0}} \varphi_{k}(\sqrt{L}) f(x)\right|>t\right\}\right| d t \\
& \leq c\|f\|_{F_{p_{0}, \infty}^{s_{0}}}^{p_{0}}=c,
\end{aligned}
$$

where we used the facts that $p_{0}<p_{1}, t \leq c^{\prime}$ and we changed the variable in the integral.

We next bound $I_{2}$. Let $t>\left(2 C^{\star}\right)^{1 / r}$. We denote by $K$ the unique integer such that

$$
C^{\star} 2^{K \frac{d}{p_{1}} r} \leq \frac{t^{r}}{2}<C^{\star} 2^{(K+1) \frac{d}{p_{1}} r} .
$$

( $K \geq 0$ necessarily). Applying now (3.6), (3.7) we extract

$$
\begin{aligned}
\sum_{k=0}^{\infty}\left(2^{k s_{1}}\left|\varphi_{k}(\sqrt{L}) f(x)\right|\right)^{r} & \leq C^{\star} 2^{K \frac{d}{p_{1}} r}+c 2^{K\left(s_{1}-s_{0}\right) r} \sup _{k \geq 0}\left|2^{k s_{0}} \varphi_{k}(\sqrt{L}) f(x)\right|^{r} \\
& \leq \frac{t^{r}}{2}+c 2^{K\left(s_{1}-s_{0}\right) r} \sup _{k \geq 0}\left|2^{k s_{0}} \varphi_{k}(\sqrt{L}) f(x)\right|^{r} .
\end{aligned}
$$

It follows that

$$
\begin{aligned}
\left|\left\{x \in \mathcal{M}:\left(\sum_{k=0}^{\infty}\left(2^{k s_{1}}\left|\varphi_{k}(\sqrt{L}) f(x)\right|\right)^{r}\right)^{1 / r}>t\right\}\right| & \leq\left|\left\{x \in \mathcal{M}: \sup _{k \geq 0}\left|2^{k s_{0}} \varphi_{k}(\sqrt{L}) f(x)\right|>c t 2^{K\left(s_{0}-s_{1}\right)}\right\}\right| \\
& \leq\left|\left\{x \in \mathcal{M}: \sup _{k \geq 0}\left|2^{k s_{0}} \varphi_{k}(\sqrt{L}) f(x)\right|>c t^{p_{1} / p_{0}}\right\}\right|,
\end{aligned}
$$

where we used the right hand inequality in (3.10) and (3.2).

We replace this expression in $I_{2}$ and by changing to the variable $\tau=t^{p_{1} / p_{0}}$ we obtain

$$
\begin{aligned}
I_{2} & \leq c \int_{0}^{\infty} t^{p_{1}-1}\left|\left\{x \in \mathcal{M}: \sup _{k \geq 0}\left|2^{k s_{0}} \varphi_{k}(\sqrt{L}) f(x)\right|>c t^{p_{1} / p_{0}}\right\}\right| d t \\
& \leq c \int_{0}^{\infty} \tau^{p_{0}-1}\left|\left\{x \in \mathcal{M}: \sup _{k \geq 0}\left|2^{k s_{0}} \varphi_{k}(\sqrt{L}) f(x)\right|>c \tau\right\}\right| d \tau \\
& =c\|f\|_{F_{p_{0}, \infty}^{s_{0}}}^{p_{0}}=c .
\end{aligned}
$$

From (3.8), (3.9) and (3.11) we establish that there exists a constant $c>0$ such that for every $f \in F_{p_{0}, \infty}^{s_{0}}$ with $\|f\|_{F_{p_{0}, \infty}^{s_{0}}}=1$,

$$
\|f\|_{F_{p_{1}, r}^{s_{1}}} \leq c
$$


Finally the embedding (3.1) follows by replacing in (3.12) $f$ by $f /\|f\|_{F_{p_{0}, \infty}^{s_{0}}}$ in the general case where $\|f\|_{F_{p_{0}, \infty}^{s_{0}}} \neq 0$. This completes the proof of the theorem.

A class of independent interest that fall under the scale of Triebel-Lizorkin spaces, is the one of (fractional) Sobolev spaces. Let $s \in \mathbb{R}$ and $p \in(1, \infty)$. A distribution $f \in \mathcal{S}^{\prime}$ belongs to the (generalized) sobolev space $H_{p}^{s}(L)$ when

$$
\|f\|_{H_{p}^{s}}:=\left\|(I+L)^{s / 2} f\right\|_{p}<\infty .
$$

It has been proved in [23] that $H_{p}^{s}=F_{p, 2}^{s}$, for every $s \in \mathbb{R}$ and $p \in(0, \infty)$. Theorem 3.1 takes the following form:

Corollary 3.2 Let $s_{0}, s_{1} \in \mathbb{R}$ and $p_{0}, p_{1} \in(0, \infty)$ be such that $s_{0}>s_{1}$ and $p_{0}<p_{1}$. Then

$$
H_{p_{0}}^{s_{0}} \hookrightarrow H_{p_{1}}^{s_{1}}, \quad \text { provided } \frac{s_{0}-s_{1}}{d}=\frac{1}{p_{0}}-\frac{1}{p_{1}}
$$

\section{Embeddings between non-classical Triebel-Lizorkin spaces}

Nonclassical spaces, allow us to deal with anisotropic geometries, where the size of a unit ball $B(x, r)$ around a point $x$ depends not only on the radious $r$ but also on $x$. In the aforementioned framework, they were introduced by Kerkyacharian and Petrushev in [23] while studying problems related to nonlinear approximation.

Definition 4.1 Let $s \in \mathbb{R}, 0<p<\infty$ and $0<q \leq \infty$. The (non-classical) Triebel-Lizorkin space $\tilde{F}_{p q}^{s}=\tilde{F}_{p q}^{s}(L)$ is defined as the set of all $f \in \mathcal{S}^{\prime}$ such that

$$
\|f\|_{\tilde{F}_{p q}^{s}}:=\left\|\left(\sum_{k \geq 0}\left(\left|B\left(\cdot, 2^{-k}\right)\right|^{-s / d}\left|\varphi_{k}(\sqrt{L}) f(\cdot)\right|\right)^{q}\right)^{1 / q}\right\|_{L_{p}}<\infty .
$$

Above the $\ell^{q}$-norm is replaced by the sup-norm if $q=\infty$.

For being able to prove embedding theorems between non-classical Triebel-Lizorkin spaces, we shall further assume the following geometric behavior of the space.

(f) Reverse doubling volume condition: There exists a constant $c_{4}>1$ such that

$$
|B(x, 2 r)| \geq c_{4}|B(x, r)| \quad \text { for all } x \in \mathcal{M} \text { and } r>0 \text {. }
$$

(g) Nonexhausting condition: There exists a constants $0<c_{5}<\infty$ such that

$$
\sup _{x \in \mathcal{M}}|B(x, 1)| \leq c_{5}
$$

The non-exhausting condition holds trivially on $\mathbb{R}^{d}$ and on every compact space; i.e. $\mu(\mathcal{M})<\infty$.

On the other hand, the reverse doubling condition (4.2) holds always true when the space $\mathcal{M}$ is connected and enjoy the doubling property (2.1) [7]. For example, every double volume Riemannian manifold, satisfies (4.2).

From (4.2) it follows that there exist constants $c_{4}^{\prime \prime} \geq 1$ and $d^{\prime \prime}>0$ such that

$$
|B(x, \lambda r)| \geq c_{4}^{\prime \prime} \lambda^{d^{\prime \prime}}|B(x, r)| \quad \text { for } x \in \mathcal{M}, r>0 \text { and } \lambda>1
$$

The maximal value $d^{\star}>0$ of the above quantity $d^{\prime \prime}$ will be referred as the lower homogeneous dimension of $\mathcal{M}$. 
Of course the dimensions satisfy $d \geq d^{\star}>0$ and it is apparent that may differ to each other. We illustrate the two dimensions in the case of the ball in the last section of this paper.

Combining (4.3) and (4.4) we find a constant $c>0$ such that

$$
|B(x, r)| \leq c r^{d^{*}} \text { for } x \in \mathcal{M} \text { and } 0<r \leq 1 .
$$

Note that the non-exhausting condition guarantees the expectable inclusion between non-classical Triebel-Lizorkin spaces (and actually it is necessary for such an inclusion):

$$
\tilde{F}_{p, q}^{s_{0}} \subset \tilde{F}_{p, q}^{s_{1}}, \quad \text { for every }-\infty<s_{0} \leq s_{1}<\infty, 0<p<\infty, 0<q \leq \infty .
$$

Let us now present the main result of the section, which illustrates the effect of the reverse-doubling property on the embedding between Nonclassical Triebel-Lizorkin spaces.

Theorem 4.2 Let $s_{0}, s_{1} \in \mathbb{R}, p_{0}, p_{1} \in(0, \infty)$ and $q, r \in(0, \infty]$ be such that $s_{0}>s_{1}$ and $p_{0}<p_{1}$. Then

$$
\tilde{F}_{p_{0}, q}^{s_{0}} \hookrightarrow \tilde{F}_{p_{1}, r}^{s_{1}},
$$

provided

$$
\frac{s_{0}-s_{1}}{d}=\frac{d}{d^{\star}}\left(\frac{1}{p_{0}}-\frac{1}{p_{1}}\right)
$$

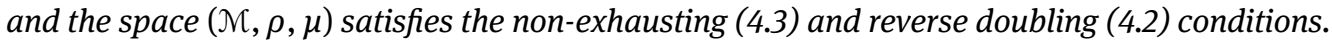

Proof. We shall follow the proof of Theorem 3.1, providing only the differences in the approach.

Let $f \in \tilde{F}_{p_{0}, \infty}^{s_{0}}$ be such that $\|f\|_{\tilde{F}_{p_{0}, \infty}^{s_{0}}}=1$. If $k \in \mathbb{N}_{0}$ by Nikol'slii's inequality (2.15) we obtain

$$
\left\|\left|B\left(\cdot, 2^{-k}\right)\right|^{-\frac{s_{1}}{d}} \varphi_{k}(\sqrt{L}) f\right\|_{\infty} \leq c\left\|\left|B\left(\cdot, 2^{-k}\right)\right|^{\frac{s_{0}-s_{1}}{d}-\frac{1}{p_{0}}}\left|B\left(\cdot, 2^{-k}\right)\right|^{-\frac{s_{0}}{d}} \varphi_{k}(\sqrt{L}) f\right\|_{p_{0}} .
$$

Since $s_{0}>s_{1}$, the growth behavior in (4.5) implies

$$
\begin{aligned}
\left|B\left(x, 2^{-k}\right)\right|^{\frac{s_{0}-s_{1}}{d}} & \leq c 2^{-k\left(s_{0}-s_{1}\right) \frac{d^{*}}{d}} \\
& \leq c 2^{-k d\left(\frac{1}{p_{0}}-\frac{1}{p_{1}}\right)},
\end{aligned}
$$

in light of the assumption (4.7).

On the other hand by (2.7) we derive

$$
\left|B\left(x, 2^{-k}\right)\right|^{-\frac{1}{p_{0}}} \leq c 2^{k d / p_{0}},
$$

which replaced in (4.8) together with (4.10) lead us to

$$
\begin{aligned}
\left\|\left|B\left(\cdot, 2^{-k}\right)\right|^{-\frac{s_{1}}{d}} \varphi_{k}(\sqrt{L}) f(\cdot)\right\|_{\infty} & \leq c 2^{k d / p_{1}}\left\|\left|B\left(\cdot, 2^{-k}\right)\right|^{-\frac{s_{0}}{d}} \varphi_{k}(\sqrt{L}) f(\cdot)\right\|_{p_{0}} \\
& \leq c 2^{k d / p_{1}}\|f\|_{\tilde{F}_{p_{0}, \infty}^{s_{0}}}=c 2^{k d / p_{1}} .
\end{aligned}
$$

Using this last inequality and similarly to (3.6) there exists a constant $\tilde{C}^{\star}>0$ such that for every integer $K \in \mathbb{N}_{0}$

$$
\left.\left.\sum_{k=0}^{K}|| B\left(x, 2^{-k}\right)\right|^{-\frac{s_{1}}{d}} \varphi_{k}(\sqrt{L}) f(x)\right|^{r} \leq c \sum_{k=0}^{K} 2^{k \frac{d}{p_{1}}}\|f\|_{\tilde{F}_{p_{0}, \infty}^{s_{0}}} \leq \tilde{C}^{\star} 2^{K \frac{d}{p_{1}} r} .
$$

Furthermore,

$$
\begin{aligned}
\left.\left.\sum_{k=K+1}^{\infty}|| B\left(x, 2^{-k}\right)\right|^{-\frac{s_{1}}{d}} \varphi_{k}(\sqrt{L}) f(x)\right|^{r} & \leq\left.\left. c \sup _{k \geq 0}|| B\left(x, 2^{-k}\right)\right|^{-\frac{s_{0}}{d}} \varphi_{k}(\sqrt{L}) f(x)\right|^{r} \sum_{k=K+1}^{\infty}\left|B\left(x, 2^{-k}\right)\right|^{\frac{s_{0}-s_{1}}{d} r} \\
& \leq\left.\left. c \sup _{k \geq 0}|| B\left(x, 2^{-k}\right)\right|^{-\frac{s_{0}}{d}} \varphi_{k}(\sqrt{L}) f(x)\right|^{r} \sum_{k=K+1}^{\infty} 2^{-k\left(s_{0}-s_{1}\right) \frac{d^{*}}{d} r} \\
& \leq\left.\left. c 2^{-K\left(s_{0}-s_{1}\right) \frac{d^{*}}{d} r} \sup _{k \geq 0}|| B\left(x, 2^{-k}\right)\right|^{-\frac{s_{0}}{d}} \varphi_{k}(\sqrt{L}) f(x)\right|^{r},
\end{aligned}
$$


thanks to (4.9) and the fact that $s_{0}>s_{1}$.

Having established inequalities (4.11) and (4.12) the remainder of the proof is identical to the one of Theorem 3.1; we omit the details.

\section{Embeddings between Besov spaces}

Let us now provide some embeddings on Besov spaces. We first recall their definition [7, 23].

Definition 5.1 Let $s \in \mathbb{R}$ and $0<p, q \leq \infty$.

(i) The Besov space $B_{p q}^{S}=B_{p q}^{S}(L)$ is defined as the set of all $f \in \mathcal{S}^{\prime}$ such that

$$
\|f\|_{B_{p q}^{s}}:=\left(\sum_{k \geq 0}\left(2^{s k}\left\|\varphi_{k}(\sqrt{L}) f(\cdot)\right\|_{L_{p}}\right)^{q}\right)^{1 / q}<\infty .
$$

(ii) The (non-classical) Besov space $\tilde{B}_{p q}^{s}=\tilde{B}_{p q}^{s}(L)$ is defined as the set of all $f \in \mathcal{S}^{\prime}$ such that

$$
\|f\|_{\tilde{B}_{p q}^{s}}:=\left(\sum_{k \geq 0}\left(\left\|\left.B\left(\cdot, 2^{-k}\right)\right|^{-s / d} \varphi_{k}(\sqrt{L}) f(\cdot)\right\|_{L_{p}}\right)^{q}\right)^{1 / q}<\infty .
$$

Above we use the standard modification when $q=\infty$.

The following embedding has been proved in [23]: Let $s_{0}, s_{1} \in \mathbb{R}, p_{0}, p_{1} \in(0, \infty]$ and $q_{0}, q_{1} \in(0, \infty]$ be such that $s_{0} \geq s_{1}, p_{0} \leq p_{1}$ and $q_{0} \leq q_{1}$. Then

$$
B_{p_{0}, q_{0}}^{s_{0}} \hookrightarrow B_{p_{1}, q_{1}}^{s_{1}}, \quad \text { and } \quad \tilde{B}_{p_{0}, q_{0}}^{s_{0_{0}}} \hookrightarrow \tilde{B}_{p_{1}, q_{1}}^{s_{1}} \quad \text { if } \quad \frac{s_{0}-s_{1}}{d}=\frac{1}{p_{0}}-\frac{1}{p_{1}} .
$$

Note that for the right side embedding, the noncollapsing condition is not required.

Assuming now that $\frac{s_{0}-s_{1}}{d}>\frac{1}{p_{0}}-\frac{1}{p_{1}}$ we can extend the range of these results including the case $q_{0}>q_{1}$. In particular we prove the following:

Theorem 5.2 Let $s_{0}, s_{1} \in \mathbb{R}, p_{0}, p_{1} \in(0, \infty]$ and $q_{0}, q_{1} \in(0, \infty]$ be such that $s_{0}>s_{1}, p_{0}<p_{1}, q_{0}>q_{1}$ and

$$
\frac{s_{0}-s_{1}}{d}>\frac{1}{p_{0}}-\frac{1}{p_{1}} \text {. }
$$

Then

(i) $B_{p_{0}, q_{0}}^{S_{0}} \hookrightarrow B_{p_{1}, q_{1}}^{S_{1}}$ and

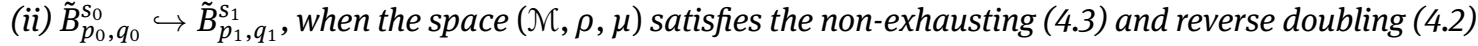
conditions.

Proof. We shall prove only claim (ii), the proof of claim (i) is similar.

Let $f \in \tilde{B}_{p_{0}, q_{0}}^{s_{0}}$. We set $\theta:=s_{0}-s_{1}-d\left(\frac{1}{p_{0}}-\frac{1}{p_{1}}\right)>0$.

By Nikol'skii's inequality (2.3) and (4.5) we obtain

$$
\begin{aligned}
\left\|\left|B\left(\cdot, 2^{-k}\right)\right|^{-\frac{s_{1}}{d}} \varphi_{k}(\sqrt{L}) f\right\|_{p_{1}} & \leq c\left\|\left|B\left(\cdot, 2^{-k}\right)\right|^{\frac{\theta}{d}-\frac{s_{0}}{d}} \varphi_{k}(\sqrt{L}) f\right\|_{p_{0}} \\
& \leq c 2^{-k \frac{d^{*}}{d} \theta}\left\|\left|B\left(\cdot, 2^{-k}\right)\right|^{-\frac{s_{0}}{d}} \varphi_{k}(\sqrt{L}) f\right\|_{p_{0}} .
\end{aligned}
$$


We putting this last inequality in the quasi-norm (5.2) and apply Hölder's inequality for the index $q_{0} / q_{1}>1$ to conclude that

$$
\begin{aligned}
\|f\|_{\tilde{B}_{p_{1}, q_{1}}^{s_{1}}} & \leq c\left(\sum_{k \geq 0}\left(2^{-k \frac{d^{*}}{d} \theta}\left\|\left|B\left(\cdot, 2^{-k}\right)\right|^{-\frac{s_{0}}{d}} \varphi_{k}(\sqrt{L}) f\right\|_{p_{0}}\right)^{q_{1}}\right)^{1 / q_{1}} \\
& \leq c\|f\|_{\tilde{B}_{p_{0}, q_{0}}^{s_{0}}}\left(\sum_{k \geq 0} 2^{-k \frac{d^{*}}{d} \theta q_{0} q_{1} /\left(q_{0}-q_{1}\right)}\right)^{\left(q_{0}-q_{1}\right) / q_{0} q_{1}} \\
& \leq c\|f\|_{\tilde{B}_{p_{0}, q_{0}}^{s_{0}}},
\end{aligned}
$$

which completes the proof of claim (ii).

Let us finally point out that claim (i) demands the non-collapsing condition (2.2), while claim (ii) demands the reverse doubling property together with the non-exhausting condition, but not the non-collapsing condition.

\subsection{Some relevant problems}

Let us finally point out that more general classes of function spaces, the so-called Besov-type and TriebelLizorkin-type spaces [33] have been recently introduced in the generality we work [25]. The embeddings between these spaces consist of an interesting open problem.

The homogeneous counterparts of Besov and Triebel-Lizorkin spaces associated with operators have been developed in $[15,16]$. The embeddings between homogeneous spaces, could be another research direction.

\section{The unit ball}

We close this paper with an example where the assumptions of our study as well as the homogeneous dimensions can be illustrated.

Let $\mathcal{M}$ be the unit ball of $\mathbb{R}^{m} ; \mathbb{B}^{m}:=\left\{x \in \mathbb{R}^{m}:\|x\|<1\right\}$, equipped with the measure

$$
d \mu(x):=\left(1-\|x\|^{2}\right)^{\gamma-1 / 2} d x, \quad \gamma>-1,
$$

and the distance

$$
\rho(x, y):=\arccos \left(\langle x, y\rangle+\sqrt{1-\|x\|^{2}} \sqrt{1-\|y\|^{2}}\right),
$$

where $\langle x, y\rangle$ is the inner product of $x, y \in \mathbb{R}^{m}$ and $\|x\|:=\sqrt{\langle x, x\rangle}$.

By [8] we have that

$$
|B(x, r)| \sim r^{m}\left(1-\|x\|^{2}+r^{2}\right)^{\gamma},
$$

which implies that $(\mathcal{M}, \mu, \rho)$ satisfies Assumption I, the reverse doubling property (4.2) and the nonexhausting condition (4.3).

The homogeneous dimensions are

$$
d=m+2 \gamma_{+} \quad \text { and } \quad d^{\star}=m+2 \gamma_{-},
$$

where $\gamma_{+}:=\max (\gamma, 0)$ and $\gamma_{-}:=\min (\gamma, 0)$.

Consider the differential operator

$$
L:=L_{\gamma}:=-\sum_{i=1}^{m}\left(1-x_{i}^{2}\right) \partial_{i}^{2}+2 \sum_{1 \leq i<j \leq m} x_{i} x_{j} \partial_{i} \partial_{j}+(m+2 \gamma) \sum_{i=1}^{m} x_{i} \partial_{i} .
$$


Then $L$ satisfies Assumption II, as it has been proved in $[8,24]$.

Acknowledgement: The authors would like to express their gratitude to the two anonymous referees, for their careful reading of the manuscript and the suggestions they offered to the authors.

The authors were funded by the University of Cyprus; Grant "NLAP-2019".

\section{References}

[1] H-Q Bui, T. A. Bui, X. T. Duong, Weighted Besov and Triebel-Lizorkin spaces associated with operators and applications. Forum Math. Sigma 8 (2020), e11, 95 pp.

[2] I. Castillo, G. Kerkyacharian, D. Picard, Thomas Bayes' walk on manifolds. Probab. Theory Relat. Fields 158, No. 3-4, 665-710 (2014).

[3] J. Cao and A. Grigor'yan, Heat kernels and Besov spaces associated with second order divergence form elliptic operators. J. Fourier Anal. Appl. 26 (2020), no. 1, Paper No. 3.

[4] J. Cao and A. Grigor'yan, Heat kernels and Besov spaces on metric measure spaces. preprint.

[5] G. Cleanthous, A. G. Georgiadis, G. Kerkyacharian, P. Petrushev, D. Picard, Kernel and wavelet density estimators on manifolds or more general metric spaces. Bernoulli 26, No. 3, 1832-1862 (2020).

[6] R. Coifman, G. Weiss, Extensions of Hardy spaces and their use in analysis. Bull. Amer. Math. Soc. 83 (1977), no. 4, $569-645$.

[7] T. Coulhon, G. Kerkyacharian, and P. Petrushev. Heat kernel generated frames in the setting of Dirichlet spaces. J. Fourier Anal. Appl., 18(5):995-1066, 2012.

[8] F. Dai, Y. Xu, Approximation theory and harmonic analysis on spheres and balls. Springer Monographs in Mathematics, Springer (2013).

[9] S. Dekel, G. Kerkyacharian, G. Kyriazis, and P. Petrushev. Compactly supported frames for spaces of distributions associated with nonnegative self-adjoint operators. Studia Math., 225(2):115-163, 2014.

[10] S. Dekel, G. Kerkyacharian, G. Kyriazis, and P. Petrushev. Hardy spaces associated with non-negative self-adjoint operators. Studia Math., 239(1): 17-54, 2017.

[11] M. Frazier, B. Jawerth, Decomposition of Besov Spaces, Indiana Univ. Math. J. 34 (1985), 777-799.

[12] M. Frazier, B. Jawerth, The $\varphi$-transform and applications to distribution spaces, Function spaces and applications (Lund, 1986), 223-246, Lecture Notes in Math., 1302, Springer, Berlin, 1988.

[13] M. Frazier, B. Jawerth, A discrete transform and decompositions of distribution, J. of Funct. Anal. 93 (1990), 34-170.

[14] M. Frazier, B. Jawerth, and G. Weiss, Littlewood-Paley theory and the study of function spaces, CBMS 79 (1991), AMS.

[15] A. G. Georgiadis, G. Kerkyacharian, G. Kyriazis and P. Petrushev, Homogeneous Besov and Triebel-Lizorkin spaces associated to non-negative self-adjoint operators. J. Math. Anal. Appl., 449 No 2 (2017), 1382-1412.

[16] A. G. Georgiadis, G. Kerkyacharian, G. Kyriazis and P. Petrushev, Atomic and molecular decomposition for homogeneous spaces of distributions associated to non-negative self-adjoint operators. Journ. Fourier Anal. Appl. (2019) 25(6), 32593309

[17] A. G. Georgiadis and M. Nielsen. Pseudodifferential operators on spaces of distributions associated with non-negative self-adjoint operators. J. Fourier Anal. Appl., 23(2):344-378, 2017.

[18] A. G. Georgiadis and M. Nielsen. Spectral multipliers on spaces of distributions associated with non-negative self-adjoint operators. J. Approx. Theory, 234 (2018), 1-19.

[19] A. Grigor'yan, and L. Liu, Heat kernel and Lipschitz-Besov spaces. Forum Math. (2014).

[20] S. Hofmann, G. Lu, D. Mitrea, M. Mitrea, and L. Yan, Hardy spaces associated to non-negative self-adjoint operators satisfying Davies-Gaffney estimates, Mem. Amer. Math. Soc. 214 (2011), no. 1007.

[21] R. Jiang and D. Yang, Orlicz-Hardy spaces associated with operators satisfying Davies-Gaffney estimates, Commun. Contemp. Math. 13 (2011), no. 2, 331-373

[22] G. Kerkyacharian, S. Ogawa, P. Petrushev and D. Picard, Regularity of Gaussian processes on Dirichlet spaces, Constr. Approx. (2018), 47:277.

[23] G. Kerkyacharian, P. Petrushev, Heat kernel based decomposition of spaces of distributions in the framework of Dirichlet spaces. Trans. Amer. Math. Soc. 367 (2015), 121-189.

[24] G. Kerkyacharian, P. Petrushev, Y. Xu, Gaussian bounds for the weighted heat kernels on the interval, ball and simplex. Constr. Approx., 51 (2020), 73-122.

[25] L. Liu, D. Yang, W. Yuan, Besov-type and Triebel-Lizorkin-type spaces associated with heat kernels. Collect. Math. 67 (2016), no. 2, 247-310.

[26] D. Müller and D. Yang, A difference characterization of Besov and TriebelLizorkin spaces on RD-spaces, Forum Math. 21 (2009), no. 2, 259-298.

[27] F. J. Narcowich, P. Petrushev, and J. D. Ward, Decomposition of Besov and Triebel-Lizorkin spaces on the sphere, J. Funct. Anal. 238 (2006), 530-564. 
[28] J. Peetre, New thoughts on Besov spaces, Duke Univ. Math. Series, Duke University, Durham, NC, 1976.

[29] E. Stein, Harmonic analysis: real-variable methods, orthogonality, and oscillatory integrals, Princeton University Press, Princeton, NJ, 1993.

[30] H. Triebel, Theory of function spaces, Monographs in Math. Vol. 78, Birkhäuser, Verlag, Basel, 1983.

[31] D. Yang, and W. Yuan, A new class of function spaces connecting Triebel-Lizorkin spaces and Q spaces. J. Funct. Anal. 255, 2760-2809 (2008).

[32] D. Yang and Y. Zhou, New properties of Besov and Triebel-Lizorkin spaces on RD-spaces, Manuscripta Math. 134 (2011), no. 1-2, 59-90.

[33] W. Yuan, W. Sickel, and D. Yang, Morrey and Campanato Meet Besov, Lizorkin and Triebel. Springer Lecture notes in mathematics, 2005, (2010). 